\title{
Choosing wisely: should past medical decisions impact the allocation of scarce ECMO resources?
}

Stephanie Kukora (skukora@med.umich.edu) ${ }^{1,2}$, Naomi Laventhal ${ }^{1,2}$

1.University of Michigan Division of Neonatal-Perinatal Medicine, Department of Pediatrics, Ann Arbor, MI, USA

2.Center for Bioethics and Social Sciences in Medicine, University of Michigan, Ann Arbor, MI, USA

\section{Correspondence}

Stephanie Kukora, Division of Neonatal-Perinatal Medicine, Department of Pediatrics, Mott Children's

Hospital, University of Michigan Medical Center,

1540 E. Hospital Dr, SPC 4254 Ann Arbor, MI

48109-4254, USA.

Tel: +734-763-4109

Fax: +734-763-7728

Email: skukora@med.umich.edu

\section{Received}

11 November 2015; revised 31 March 2016;

accepted 2 May 2016

DOI:10.1111/apa.13457

Clinical trial registration: None

On a cold night in January 2013, I checked my email and was surprised by the message: 'Due to the H1N1 flu epidemic, we cannot support any further ECMO patients as we have reached the limit of our equipment and staffing'. My thoughts went to the expectant mother upstairs, carrying a fetus with an anomaly whose only hope for survival was ECMO. I wondered how I would tell her that the life-support therapy her baby needed would not be available if she delivered tonight, knowing that this resource had been exhausted by patients with potentially preventable illness.

We argue treating ECMO as a scarce resource analogous to donated organs allows for a more prudent approach to its allocation. A policy of withholding ECMO from patients who have refused the influenza vaccine serves two functions: further incentivising vaccination compliance and more justly allocating scarce resources in times of crisis. Ethical

Articles in the series A Different View are edited by William Meadow (wlm1@uchicago.edu).We encourage you to offer your own different view either in response to A Different View you do not fully agree with, or on an unrelated topic. Send your article to Dr. Meadow (wlm1@uchicago.edu).

Abbreviations

ECMO, Extracorporeal membrane oxygenation.
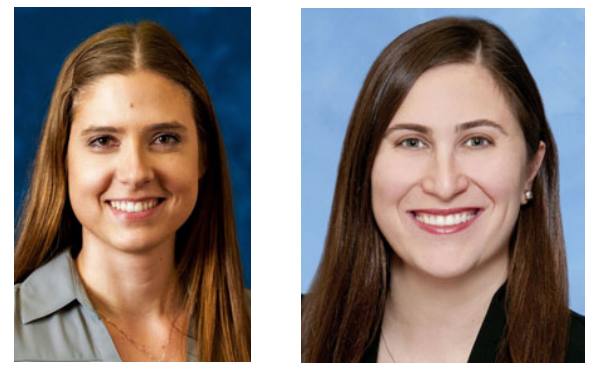

implementation would necessitate widespread education regarding existence of the policy so that those continuing to refuse the vaccine are giving 'informed refusal'. Requiring patients who refuse the vaccine during a routine office visit to sign a waiver demonstrating understanding that they may be ineligible for this intervention is one way of accomplishing this. Enacting the policy only when certain criteria are met, such as influenza pandemic or other crisis situations, allows for discretionary and compassionate utilisation of ECMO in the absence of overriding demand.

\section{ECMO}

Extracorporeal membrane oxygenation (ECMO) is a lifesustaining therapy for patients with severe cardiorespiratory failure. It is a limited resource available in a few hundred centres in the United States, each with a fixed number of circuits. As of 2011, nearly half of patients who received ECMO were newborns with respiratory failure. This is the population for whom ECMO was developed and still has the best reported outcomes (1). Indications have expanded to include paediatric and adult patients with other causes of cardiorespiratory failure; average survival in these age groups is considerably lower, although the risk/benefit ratio and prognosis is variable and based on individual factors. Its use is considered cost-effective for adults and children with reversible disease $(2,3)$.

The frequency and severity of complications limits ECMO only to patients with high risk of imminent death. 
It is considered when mortality risk approaches 50\% and indicated when it is anticipated to exceed $80 \%$ (1). It is typically allocated among eligible patients using a 'firstcome, first-served' rule. Within ECMO centres, a fixed number of circuits are available, limited by equipment, specialists who continually oversee it, and blood products. Although transport to other centres can be helpful in localised shortage, transport may be risky or entirely unfeasible, and all centres may be at capacity during pandemics and other widespread crises.

\section{INFLUENZA}

Influenza is a prevalent illness with significant morbidity and mortality. In 2009, the H1N1 strain caused aggressive respiratory failure, leading to investigation of ECMO as a rescue therapy for older children and adults (4). Subsequently, H1N1 vaccine development offered an effective prevention strategy. During the 2013-2014 influenza seasons, an estimated 7.2 million cases were prevented by vaccination, resulting in 90000 fewer hospitalisations (5).

The influenza vaccine is safe and cost-effective. Insurance coverage is mandated by the Affordable Care Act, and it is available outside of the doctor's office, which may lower the cost of administration (6). Nonetheless, fewer than half of eligible patients received the influenza vaccine during the 2013-2014 season (7), and a regional study noted the majority of patients who required intensive care from influenza were unvaccinated (8). Reasons for nonvaccination include lack of access to preventative health care, philosophical objection and, importantly, misperceptions about the mechanism or side effects - for example belief that vaccination causes 'the flu' or that the vaccine symptoms are worse than influenza infection (9). These misperceptions may, in part, be attributable to framing. When obtaining informed consent, physicians must discuss potential risks of the vaccine but not the risks of declining vaccination (10). This encourages 'omission bias', in which consequences of action (experiencing a complication of influenza vaccine) are judged more harmful than consequences of inaction (experiencing a complication of influenza).

\section{THE ETHICAL DILEMMA}

There is a prima facie duty to treat patients with every resource regardless of personal decisions they made prior to becoming ill. This duty came to the national consciousness with the publication in Life magazine of the story of the Seattle 'God Committee' which determined allocation of the few dialysis machines available, often utilising 'social utility' criteria. Distribution of scarce resources is a longstanding topic of ethical debate(11), and healthcare rationing strategies are often considered ethically suspect. Criteria such as age, social standing and instrumental value of the patient seem discriminatory in our egalitarian society. The first-come, first-served model is effective in situations requiring rapid decision-making; little needs to be known about the patient and each patient is provided equality of opportunity. Unfortunately, this approach inadequately addresses the concept of fairness, as an infant deprived of ECMO has more potential and years of life ahead of her than an adult who may have already lived half or more of her life and could not have opted for a safe and efficacious preventative intervention.

A possible solution to the dilemma of ECMO shortage in H1N1 pandemic is to withhold ECMO for influenza complications among those who declined vaccination. This could be achieved by developing and publicising a policy making vaccine refusal a criteria used in ECMO triage decisions in crisis situations, and having competent adult patients sign a waiver acknowledging their understanding of this policy when declining immunisation. Vaccinated adults, minors, those with medical contraindications to vaccination, without access to preventative health care, or not competent to make decisions would retain access.

Is this ethically permissible? Historically, past decisions have been considered in allocation of limited health resources. Solid organs for transplantation are a limited resource for which demand greatly outweighs supply, and some have argued that patients with alcoholic liver disease should be deprioritised (12). Although no position is endorsed by the United Network for Organ Sharing, most transplant centres will not list alcoholic patients unless they comply with designated abstinence criteria. Reasons for abstinence criteria are both medical and ethical, as it improves transplant success and reassures decision-makers that the organs are not misallocated. This has been criticised by those who argue that continued alcohol use is not a choice but a manifestation of underlying disease. Vaccine refusal, however, is not an illness.

The question becomes whether it is morally acceptable to deny a patient a potentially life-saving therapy in order to reserve the resource for another anticipated to need it, not whether unvaccinated adults are deserving of life-saving health care for severe complications of influenza. If adequate resources existed, denying a patient desired ECMO therapy on the basis of vaccination status would be impermissible and violate the principles of beneficence and nonmaleficence; physicians should not be arbiters of medical care or pass judgement on patients based on past decisions. However, currently and foreseeably, ECMO remains a limited resource. In shortage, different principles come into play: autonomy and justice. The question becomes, 'are patients entitled to make decisions about their own health care against physician recommendation of if those decisions imperil others?'

We acknowledge that the impracticalities of instating such a waiver are significant: some patients do not decline vaccination, but rather are never offered it; others may refuse both vaccination and the waiver. Enforcement would also be difficult - determining the degree of shortage warranting ECMO rationing, and consistently applying allocation schemes would be complex and morally distressing. But we propose this as an approach to shifting the nature of informed 
consent for influenza vaccine away from a neutrally offered option whose refusal is respected as equally valid as acceptance. Although some patients thoughtfully decline vaccination based on value systems that weigh medical interventions as worse than illness or death, many simply lack understanding of Bayesian probabilities and have misplaced fears and miscalculated risks. It seems illogical to desire the risk/benefit profile of ECMO therapy as treatment for life-threatening respiratory failure, but not that of vaccination for prevention of influenza altogether. Engaging patients in a discussion which requires them to view their decisions not only in the context of public health, but also in personal consequences of opting out, may lead to reconsideration of risk assessment about future events and acceptance of vaccination. It also may clarify values of noninterventionists who truly desire neither vaccination nor ECMO.

Our proposal withstands analysis within multiple ethical frameworks. Providing therapy to patients for whom ECMO has most consistently proven effective and who have the most life-years to gain satisfies the consequentialist theory of utilitarianism. Within a nonconsequentialist framework (Principle of Fairness), a scarce resource is justly distributed, prioritising those unable to prevent their illness. And although vaccination may be viewed as desirable but not morally imperative, in agent-centred theory (Virtue Ethics), participation in herd immunity may be considered obligatory, as contribution to the health of the general population is the virtuous action.

\section{CONCLUSION}

Personal autonomy over health decisions is highly valued, but consequences of decisions may adversely affect others. The 'first-come, first-served' allocation model leads to a conflict between autonomy and distributive justice. Having those who refuse influenza vaccination waive access to future ECMO therapy for subsequent influenza complications would require competent adults to acknowledge the potential consequences of their decision, clarify values, and permit more just allocation of this limited resource.

\section{FUNDING SOURCE}

No funding was secured for this study.

\section{FINANCIAL DISCLOSURE}

The authors have no financial relationships relevant to this article to disclose.

\section{CONFLICT OF INTEREST}

The authors have no conflicts of interest to disclose.

\section{References}

1. Annich G, editor. ECMO: extracorporeal cardiopulmonary support in critical care, 4th ed. Ann Arbor: Extracorporeal Life Support Organization, 2012.

2. Peek GJ, Elbourne D, Mugford M, Tiruvoipati R, Wilson A, Allen E, et al. Randomised controlled trial and parallel economic evaluation of conventional ventilatory support versus extracorporeal membrane oxygenation for severe adult respiratory failure (CESAR). Health Technol Assess 2010; 14: $1-46$.

3. Petrou S, Edwards L. UK Collaborative ECMO Trial. Cost effectiveness analysis of neonatal extracorporeal membrane oxygenation based on four year results from the UK Collaborative ECMO Trial. Arch Dis Child Fetal Neonatal Ed 2004; 8: F263-8.

4. Zangrillo A, Biondi-Zoccai G, Landoni G, Frati G, Patroniti $\mathrm{N}$, Pesenti A, et al. Extracorporeal membrane oxygenation (ECMO) in patients with H1N1 influenza infection: a systematic review and meta-analysis including 8 studies and 266 patients receiving ECMO. Crit Care 2013; 17: R30.

5. Reed C, Kim IK, Singleton JA, Chaves SS, Flannery B, Finelli L, et al. Estimated influenza illnesses and hospitalizations averted by vaccination-United States, 2013-14 influenza season. MMWR Morb Mortal Wkly Rep 2014; 63: 1151-4.

6. Prosser LA, Lavelle TA, Fiore AE, Bridges CB, Reed C, Jain S, et al. Cost-effectiveness of 2009 pandemic influenza A (H1N1) vaccination in the United States. PLOS ONE 2011; 6: e22308.

7. Centers for Disease Control and Prevention. Flu vaccination coverage, United States, 2013-14 influenza season. Content last reviewed 18 Sept. 2014. Accessed Oct 8 2014. Available at: http://www.cdc.gov/flu/fluvaxview/coverage-1314estimates. htm.

8. Catania J, Que LG, Govert JA, Hollingsworth JW, Wolfe CR. High intensive care unit admission rate for 2013-2014 influenza is associated with a low rate of vaccination. Am J Respir Crit Care Med 2014; 189: 485-7.

9. Redelings MD, Piron J, Smith LV, Chan A, Heinzerling J, Sanchez KM, et al. Knowledge, attitudes, and beliefs about seasonal influenza and H1N1 vaccinations in a lowincome, public health clinic population. Vaccine 2012; 30: 454-8.

10. Centers for disease control and prevention. vaccine information sheet: inactivated influenza vaccine. Content posted 19 Aug. 2014. Accessed Oct. 8 2014. Available at: http://www.cdc.gov/vaccines/hcp/vis/vis-statements/flu.pdf.

11. Persad G, Wertheimer A, Emanuel EJ. Principles for allocation of scarce medical interventions. Lancet 2009; 373: 16.

12. Moss AH, Siegler M. Should alcoholics compete equally for liver transplantation? JAMA J Am Med Assoc 1991; 265: 1295-8. 